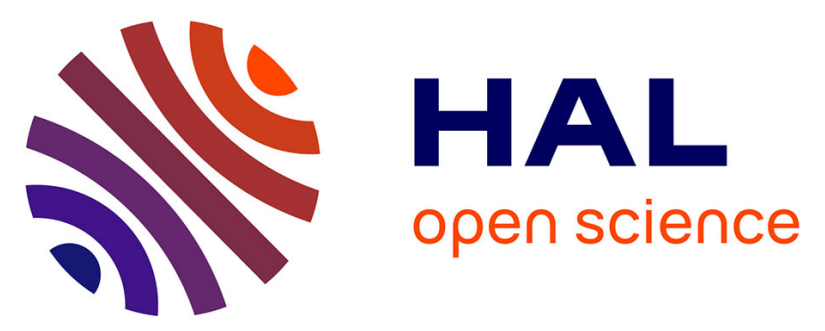

\title{
The rotational propulsion characteristics of scaled-up helical microswimmers with different heads and magnetic positioning.
}

Tiantian Xu, Gilgueng Hwang, Nicolas Andreff, Stéphane Régnier

\section{- To cite this version:}

Tiantian $\mathrm{Xu}$, Gilgueng Hwang, Nicolas Andreff, Stéphane Régnier. The rotational propulsion characteristics of scaled-up helical microswimmers with different heads and magnetic positioning.. IEEE/ASME International Conference on Advanced Intelligent Mechatroncis, AIM'13., Jan 2013, Australia. pp.1114-1120. hal-00866314

\section{HAL Id: hal-00866314 https://hal.science/hal-00866314}

Submitted on 26 Sep 2013

HAL is a multi-disciplinary open access archive for the deposit and dissemination of scientific research documents, whether they are published or not. The documents may come from teaching and research institutions in France or abroad, or from public or private research centers.
L'archive ouverte pluridisciplinaire HAL, est destinée au dépôt et à la diffusion de documents scientifiques de niveau recherche, publiés ou non, émanant des établissements d'enseignement et de recherche français ou étrangers, des laboratoires publics ou privés. 


\title{
The Rotational Propulsion Characteristics of Scaled-up Helical Microswimmers with different heads and magnetic positioning
}

\author{
Tiantian $\mathrm{Xu}^{1}$, Gilgueng Hwang ${ }^{2}$, Nicolas Andreff ${ }^{3}$ and Stéphane Régnier ${ }^{1}$
}

\begin{abstract}
Micro and nanorobots capable of controlled propulsion at low Reynolds number are foreseen to change many aspects of medicine by enabling targeted diagnosis and therapy, and minimally invasive surgery. Several kinds of helical swimmers with different heads actuated by a rotating magnetic field have been proposed in prior works. Beyond these proofs of concepts, this paper aims to study behaviours of helical swimmers with different head and magnetic positing adapted to low Reynolds number liquids. For this, we designed an experimental setup and scaled-up helical nanobelt swimmers with different heads and tail coatings to compare their rotational propulsion characteristics. We found in this paper that the head shape of a helical swimmer does not influence on the shape of the rotational propulsion characteristics curve, but it influences on the values of the cut-off frequency. The rotational propulsion characteristics of helical swimmers with a magnetic head or a magnetic tail are much different. The helical swimmer with uniformly coated magnetic tail does not show a cut-off frequency but a saturation of frequency.
\end{abstract}

\section{INTRODUCTION}

Micro and nanorobots have a great impact in medicine. Microrobots can be used for the localized delivery of chemical and biological substances, to remove material by mechanical means, to act as simple controllable static structures or to transmit biological data from a specific hard to reach location [1].

The medical context often implies that the micro and nanorobots have to swim in fluid. However, at the micro and nano scale, the fluid become extremely viscous and the Reynolds number of the micro and nanorobots dramatically lower. Purcell described two swimming techniques which are suitable for swimming at low Reynolds numbers [2], [3] : corkscrew type rotating propulsion and oscillation of flagella. Micro and nanoscale helical propellers are inspired by E. coli bacteria. E.coli bacteria consist of a rod-shaped head and a bundle of passive helical flagella. Flagella are driven by a rotary motor to generate a corkscrew-like motion [4].

Since more than ten years ago, researchers have developed several different micro helical swimmers, specially with different head shapes. The first helical

\footnotetext{
${ }^{1}$ T.XU and S.Régnier are with ISIR - Insti-
tut des Systèmes Intelligents et Robotique, UPMC, Paris, France. tiantian.xulisir.upme.fr, stephane.regnier@upmc.fr

${ }^{2} \mathrm{G}$. Hwang is with Laboratoire de Photonique et de Nanostructures, Marcoussis, France. gi lgueng.hwang@lpn.cnrs. fr

${ }^{3} \mathrm{~N}$. Andreff is with FEMTO-ST Institute, UFC, Besancon, France. nicolas.andreffefemto-st.fr
}

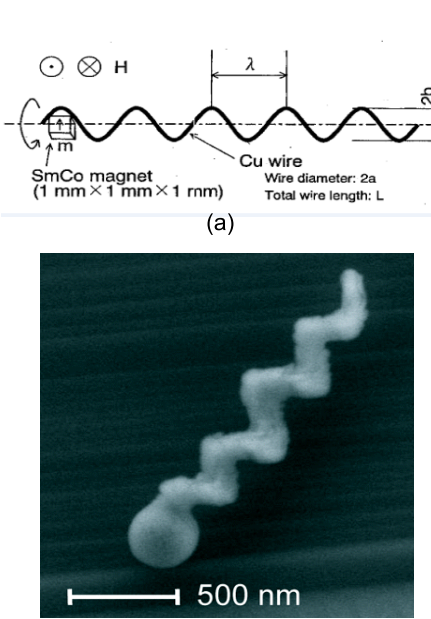

(c)

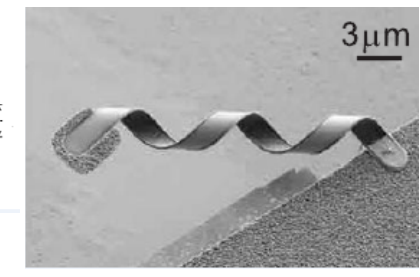

(b)

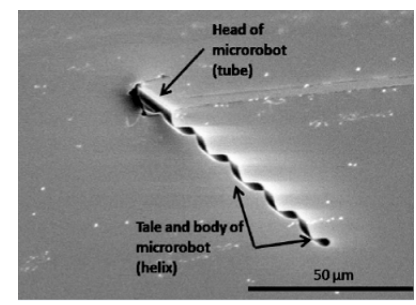

(d)

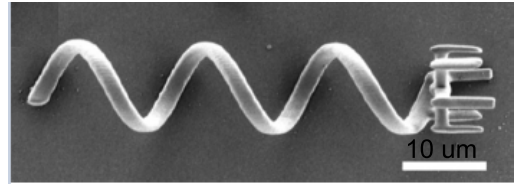

(e)

Fig. 1: (a) The first helical type swimmer with a cubic magnetic head [5]. (b) Artificial Bacterial Flagella with a thin-square-plate soft magnetic head [6], [7]. (c) A smaller helical swimmer with a spherical head [8]. (d) Helical Nanobelt with a cylindrical tube head [9], [10]. (e) A polymer helical swimmer with a "claw" shaped holder head [11].

type swimming machine was proposed by Honda et al. in 1996 [5], as shown in Fig. 1a. This swimming machine was as large as a few millimetres, with a strong permanent magnetic cubic head and a helical tail made of copper filament. This device could be driven wirelessly by an external rotating magnetic field. The swimming performance tests were in low Reynold number conditions. They predicted that this helical type swimming machine with a length of $11.5 \mathrm{~mm}$ could still be scaled down to micrometer-sized machine [12]. Thanks to the recent development in micro and nanotechnology, researchers of ETH Zurich fabricated microscale helical swimmers in 2007, called "Artificial Bacterial Flagella" (ABF), by using a self-scrolling fabrication technique [13], [6], [14], [7]. The ABF consisted of a helical tail made by GaAs/InGaAs and a thinsquare-plate $(4.5 \mu \mathrm{m} \times 4.5 \mu \mathrm{m} \times 200 \mathrm{~nm})$ soft magnetic 
head on one end, as shown in Fig. 1b. The total length of the ABF was approximately $50 \mu \mathrm{m}$ and its thickness was approximately $30 \mathrm{~nm}$. A group at Harvard presented in 2009 even smaller helical swimmers with a diameter of $200 \mathrm{~nm}$ and a length of $2 \mu \mathrm{m}$ [8]. The swimmers were made of glass $\left(\mathrm{SiO}_{2}\right)$. The swimmers have spherical heads. A permanent magnetic film was evaporated onto one side of the swimmers, as shown in Fig. 1c. Our group at ISIR showed that electro-osmosis propulsion was more efficient than magnetically actuated propulsion [9], [10]. The propulsion achieved a speed of 24 body lengths per second. The micro helical swimmers we used, called "Helical NanoBelt" (HNB), had a cylindrical head and a helical tail. The total length was about $70 \mu \mathrm{m}$. The entire surface was coated by a $10 \mathrm{~nm}$ thick Nickel layer. This surface-coated HNBs, as shown in Fig. 1d, has a similar geometry to ABF, but their entire surface can be functional to propulsion in comparison to the ABF's soft magnetic head as the only functional part and the tail as a passive part. More recently, the ETH group presented a helical swimmer with a "claw" shaped holder attached to a polymer helical tail, as shown in Fig. 1e [11]. This holder was used to transport micro objects.

So far, several different heads for helical swimmers have appeared. However their influence on propulsion has not been shown yet. Moreover, the difference between the propulsion behaviours of helical swimmers with a magnetic head or with a magnetic tail has not been clarified. We started by studying the rotational propulsion characteristics of different helical swimmers in this paper. We first proposed to fabricate scaled-up helical swimmers with different head shapes to compare their rotational propulsion characteristics. Then, the rotational propulsion characteristics between helical swimmers with magnetic heads and those with magnetic tails were compared. Two methods were used to make a magnetic tail. One method consists in covering the helical tail with small magnets, the other one in uniformly coating the helical tail in magnetic material. Later the rotational propulsion characteristics between the two kinds of magnetic tails were compared.

Section II introduces the different types of scaledup helical nanobelt, viscous liquids used in the paper and the magnetic actuating system. Section III presents the modelling of the helical swimmers motion with a magnetic head or with a magnetic tail. Section IV describes the rotational propulsion characteristics of different helical swimmers.

\section{EXPERIMENTAL SETUP}

\section{A. Scaled-up helical swimmers with different heads}

The swimming behaviour of microscale helical swimmers has not been clearly defined mainly due to the limited observation tools. Moreover, the micro fabrication process for self-rolling HNBs usually takes long. Making different head shapes and different geometries

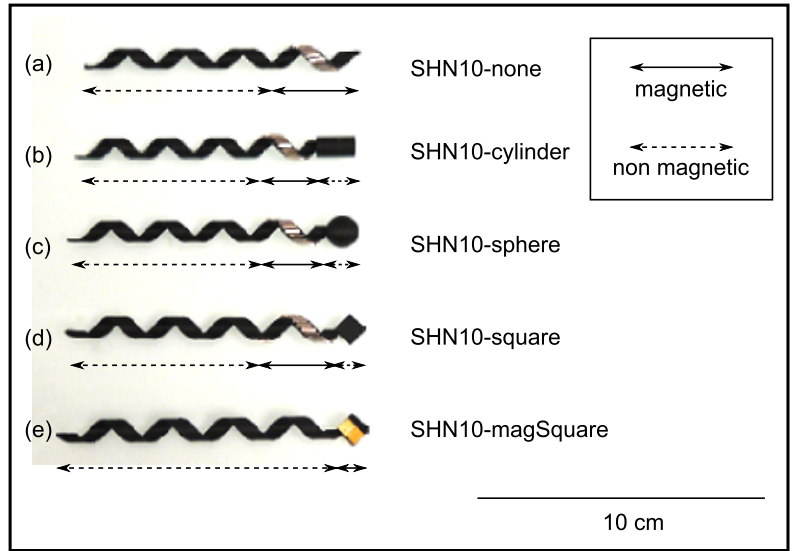

Fig. 2: The Scaled-up helical nanobelts of $10 \mathrm{~cm}$ length (SHN10s) with different heads : (a) SHN10-none : with no head. (b) SHN10-cylinder : with a cylindrical head. (c) SHN10-sphere : with a spherical head. (d) SHN10square : with a square head. (e) SHN10-magSquare : with a magnetic square head.

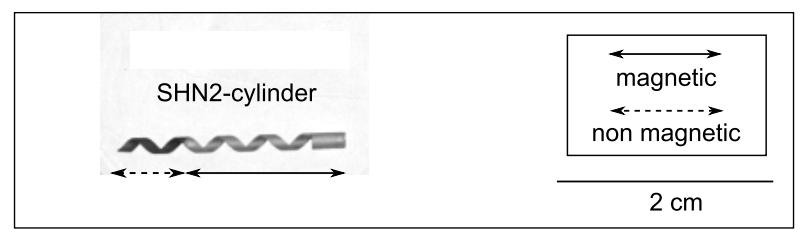

Fig. 3: The Scaled-up helical nanobelts of $2 \mathrm{~cm}$ length (SHN2s) with cylinder head.

is a challenge for micro-fabrication. That is why Scaledup Helical Nanobelts (SHNs) are designed. Two scales of SHNs were made : SHN10s and SHN2s.

1) SHN10s: SHN10s are made of polymer by a rapid prototyping method. The length of SHN10s are about $10 \mathrm{~cm}$. The geometry is scaled up proportionally except for the thickness, because the minimal thickness of the fabrication is limited. The following two categories of SHN10 were made. The category named SHN10-magTail includes SHN10s with their first pitch (i.e. a 1-full-turn portion of the helix) covered with $\mathrm{NdFeB}$ permanent magnets $(5 \mathrm{~mm} \times 1.5 \mathrm{~mm} \times$ $1 \mathrm{~mm})$, since as described in [15], the SHN10s with one magnetic pitch are more advantageous than those with full magnetic pitch. In this category, SHN10s have different head shapes : without a head, with a cylindrical head, spherical head and square head, as shown in Fig. 2. The heads are not magnetic. They are named SHN10-none, SHN10-cylinder, SHN10-sphere and SHN10-square. The other category, named SHN10magHead, includes one SHN10-magSquare with a square head covered by 4 rectangular $\mathrm{NdFeB}$ magnets $(5 \mathrm{~mm} \times 4 \mathrm{~mm} \times 1 \mathrm{~mm})$ on the two sides. The whole tail of this SHN10-magSquare is non-magnetic.

2) SHN2s: SHN2s are made from titanium. They were made by i.materialise (3D printing company). They 
TABLE I: Specifications of the HNB and the SHNs [9].

\begin{tabular}{l|c|c|c} 
Parameters & HNB & SHN10 & SHN2 \\
\hline Diameter & $2.1 \mu \mathrm{m}$ & $6 \mathrm{~mm}$ & $1.2 \mathrm{~mm}$ \\
Thickness & $27.2 \mathrm{~nm}$ & $1 \mathrm{~mm}$ & $0.2 \mathrm{~mm}$ \\
Pitch & $14 \mu \mathrm{m}$ & $20 \mathrm{~mm}$ & $4 \mathrm{~mm}$ \\
Width & $2.5 \mu \mathrm{m}$ & $7.2 \mathrm{~mm}$ & $1.44 \mathrm{~mm}$ \\
Length & $62 \mu \mathrm{m}$ & $97.2 \mathrm{~mm}$ & $17.8 \mathrm{~mm}$ \\
\hline Nb of turns & \multicolumn{3}{|c}{4.5} \\
\hline Weight & - & (a) $2.08 \mathrm{~g}$ & (a) $0.04 \mathrm{~g}$ \\
& & (b) $1.75 \mathrm{~g}$ & (b) $0.05 \mathrm{~g}$ \\
& (c) $2.63 \mathrm{~g}$ & (d) $1.85 \mathrm{~g}$ & (e) $1.33 \mathrm{~g}$ \\
\hline Magnetic material & Nickel & NdFeB & Nickel \\
Magnetic layer & $30 \mathrm{~nm}$ & $1 \mathrm{~mm}$ & $20-50 \mu \mathrm{m}$
\end{tabular}

are coated by ferromagnetic material : nickel. The length of SHN2s is about $2 \mathrm{~cm}$. SHN2s are designed smaller in order to make the coating process easier. For the thick layer coating, electroplating was used. The titanium models were placed onto the negative electrodes and the positive electrode was placed near the models. These were immersed in a nickel bath with the application of electric potential between the two electrodes. The deposition rate was calibrated onto the dummy silicon surface coated with titanium layer. The end of the helical tail is used to make an electric contact during the electrolytic deposition process, thus the last pitch of SHN2 is not coated. Table I summarizes the specifications of the two scales of SHNs.

\section{B. Swimming at low Reynolds number}

The Reynolds number is a dimensionless quantity, which is defined as the ratio of the inertial forces $\left(f_{i n}\right)$ to the viscous forces $\left(f_{v}\right)$ by Osborne Reynolds about a hundred years ago [16]. So when the Reynolds number is far below 1, the viscous forces dominate the inertial forces. The Reynolds number can be expressed as a function of the object velocity $(v)$, the object characteristic size $(L)$, the fluid density $(\rho)$ and the fluid dynamic viscosity $(\mu)$ :

$$
R e=\frac{\rho v L}{\mu}
$$

Swimming performances of microscale swimmers are largely limited due to the low Reynolds dynamics [17].

To emulate at an upper-scale the environment of microscale robots swimming at low Reynolds numbers, one can use more viscous liquid. In the following experiments, the viscous liquids used are glycerol solutions with different concentrations. Their viscosities are measured by a falling ball viscometer (Brookfield KF10). The viscosity of glycerol is sensitive to environmental temperature. The measured experimental temperature is about $23{ }^{\circ} \mathrm{C}$. Table II summarizes the measured viscosities of glycerol solutions used and the calculated $R e$ at which the two sizes of SHNs swim in the experiments. In this paper, only the rotational propulsion characteristics were studied. In order to

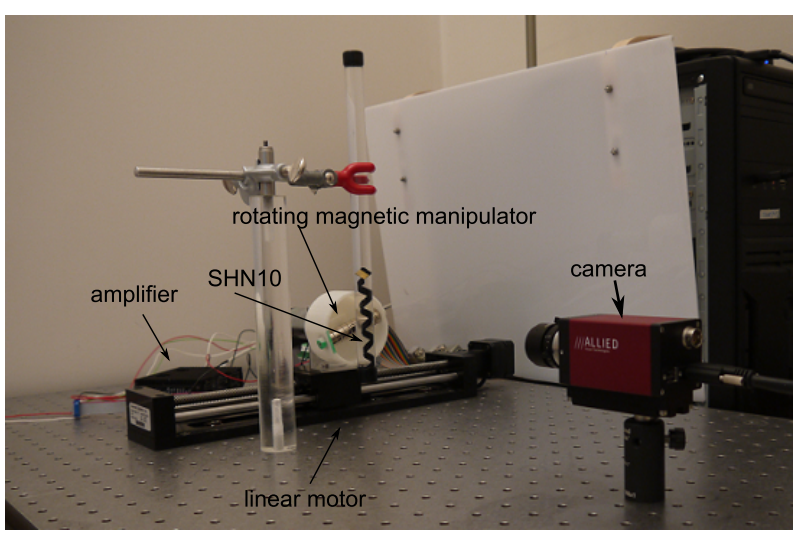

Fig. 4: Photo of the experimental setup. A SHN10 with a magnetic square head inside a transparent tube is placed before a rotating magnet manipulator.

calculated the $R e$, we assume the translational velocity was 1 pitch per second. The object characteristic size was considered as the thickness of the SHNs.

TABLE II: Measured viscosity of different glycerol solution and calculated $R e$ at which SHN10s and SHN2 swim in the experiments.

\begin{tabular}{l|llll} 
\% of Glycerol & $\begin{array}{l}\text { viscosity } \\
(\mathrm{mPa} \cdot \mathrm{s})\end{array}$ & $\begin{array}{l}\text { density } \\
\left(\mathrm{g} / \mathrm{m}^{3}\right)\end{array}$ & $\operatorname{Re}(\mathrm{SHN10})$ & $\operatorname{Re}(\mathrm{SHN2})$ \\
\hline $30 \%$ & 3.2 & 1.09 & - & 0.54 \\
$60 \%$ & 16.1 & 1.17 & 1.45 & 0.11 \\
$70 \%$ & 32.4 & 1.19 & 0.73 & 0.06 \\
$80 \%$ & 66.5 & 1.21 & 0.36 & 0.03 \\
$85 \%$ & 124.6 & 1.22 & 0.20 & - \\
$90 \%$ & 260.0 & 1.23 & 0.09 & 0.008
\end{tabular}

\section{Experimental setup}

Researchers utilized orthogonal arrangements of electromagnetic coil pairs to generate rotating uniform magnetic fields at the center of the system's workspace to propel the helical swimmers [7], [8]. However, these coil pairs are difficult to be scaled up [17]. Moreover, the energy efficiency largely decreases when we scaled them up. Instead of the coil pairs, a rotating permanent magnet allows to control our scaled-up models wirelessly [18], [19]. The magnet used in the experiments is cylindrical, $60 \mathrm{~mm}$ in length and $15 \mathrm{~mm}$ in diameter, mounted on a Maxon DC motor. The rotation frequency of the motor is controlled. The chosen configuration of the magnetic actuation is lateral. In this configuration, the SHNs can be more proximate to the magnet thus we can use smaller magnetic field. Later, the rotating magnetic field can be combined with a translational movement by linear stage. The SHNs are placed $25 \mathrm{~mm}$ away from the axis of the magnet. The magnetic field strength around the magnetic part of the SHNs is about $30 \mathrm{mT}$.

The camera used in the experiments was a PIKE F032C firewire camera. In the experiments, the rotations of the SHNs are recorded by the camera. The 
rotation frequency of the SHN is calculated off-line by the time that a SHN takes to rotate one turn. The frame rate used is 50 frames per second. The maximal rotation frequency measured in the experiments is about $20 \mathrm{~Hz}$. The rotation frequency error is estimated at about $2 \%$.

\section{Modelling}

The helical swimmers are actuated by a rotating magnetic field. All magnetized objects within an externally imposed magnetic field will have both forces and torques exerted on them. Magnetic forces $\left(\overrightarrow{f_{m}}\right)$ are proportional to the gradient of the magnetic field $(\nabla \vec{B})$ in the neighbourhood of the magnetized object, and act to move the object to local maxima. Magnetic torques $\left(\overrightarrow{\tau_{m}}\right)$ are proportional to the magnetic field $(\vec{B})$ and act to align the internal magnetization of an object $(\vec{M})$ with the field. We suppose that the magnetization of the object is uniform. The equations that express the interactions are as follows [20] :

$$
\begin{aligned}
& \overrightarrow{f_{m}}=V_{m}(\vec{M} \cdot \nabla) \vec{B} \\
& \overrightarrow{\tau_{m}}=V_{m} \vec{M} \times \vec{B}
\end{aligned}
$$

where $V_{m}$ is the volume of the magnetized object. An applied magnetic field at the location of the magnetized object transduces into torque and the spatial magnetic gradient transduces into applied force.

\section{A. Modelling of the helical swimmer with square magnetic} head

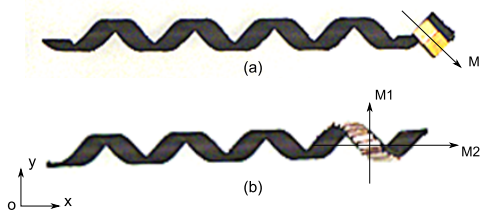

Fig. 5: (a) The magnetization (M) direction of the SHN10-magSquare is along the longer edge of the rectangular magnet on its head. (b) The helical swimmer with a magnetic tail (e.g. SHN10-none) has two directions of magnetization. M1 which is perpendicular to the $x$ axis contributes to propulsion. M2 causes the swimmer to wobble.

We first model the SHN10-magSquare with a square magnetic head. The SHN10-magSquare is put before the rotating magnet manipulator, along the $x$ axis. The magnetization (M) direction of the SHN10-magSquare is along the longer edge of the rectangular magnet on its head, as shown in Fig. 5a. The initial position of the magnet manipulator is along the $x$ axis, as shown in Fig. $6 \mathrm{a}, \mathrm{b}$ and $\mathrm{c}$. The magnetic field generated by the rotating permanent magnet manipulator is simulated by Comsol. The red arrows represent the magnetic field. According to Equation 3, the magnetic torque around the $x$ axis is zero (i.e. $\left.(\vec{M} \times \vec{B}) \cdot \overrightarrow{u_{x}}=0\right)$ at the initial position. After the magnet manipulator turns $45^{\circ}$, the magnetic field near the head position of the SHN10-magSquare turns at an angle around the $x$ axis, as shown in Fig. 6d. If the SHN10-magSquare stays in its initial position, it will be subject to a magnetic torque around the $x$ axis (i.e. $(\vec{M} \times \vec{B}) \cdot \overrightarrow{u_{x}} \neq 0$ ). Thus, the SHN10-magSquare will follow the magnetic field. The magnetic field near the head position of the SHN10magSquare projected on the plane yoz rotates a whole turn around the $x$ axis, if the magnet manipulator rotates one turn. As a result, the SHN10-magSquare rotates around the $x$ axis by the action of the rotating magnetic field.

\section{B. Modelling of the helical swimmer with magnetic tail}

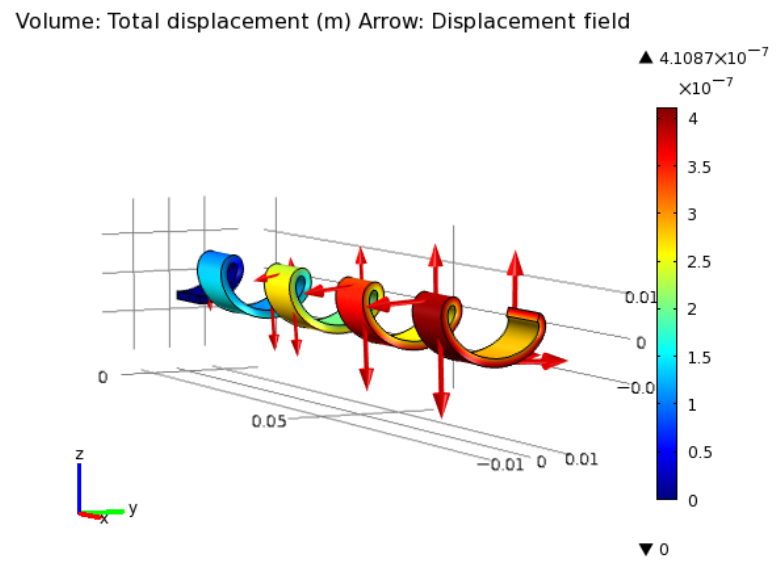

Fig. 7: The deformation simulation of a helical swimmer with a magnetic tail exerted a volumetric torque around its axis with one end fixed.

The swimmers with helical magnetic tail have two directions of magnetization. One is perpendicular to their axis (M1), the other one is along their axis (M2), as shown in Fig. 5b. M1 contributes to propelling itself. A volumetric magnetic torque exerts on the helical swimmer with a magnetic tail around the $x$ axis, according to Equation (3). A deformation of the SHN10none exerted a torque around its axis with one end fixed is simulated and presented by Fig. 7. The applied torque is volumetric. The displacement along the $x$ axis is blocked. The red arrows represent the displacements of the points at the end of the arrows. The helical swimmer is twisted. If, instead of being fixed, the head was free, the swimmer would then rotate. M2 causes the swimmer to wobble.

\section{ROTATIONAL PROPULSION CHARACTERISTICS}

\section{A. Head influence on rotational propulsion characteristics}

In this section, we designed a series of experiments to show the influence of head shapes on rotational propulsion characteristics of helical swimmers. The SHN10magTails (SHN10s with their first pitches magnetic) 

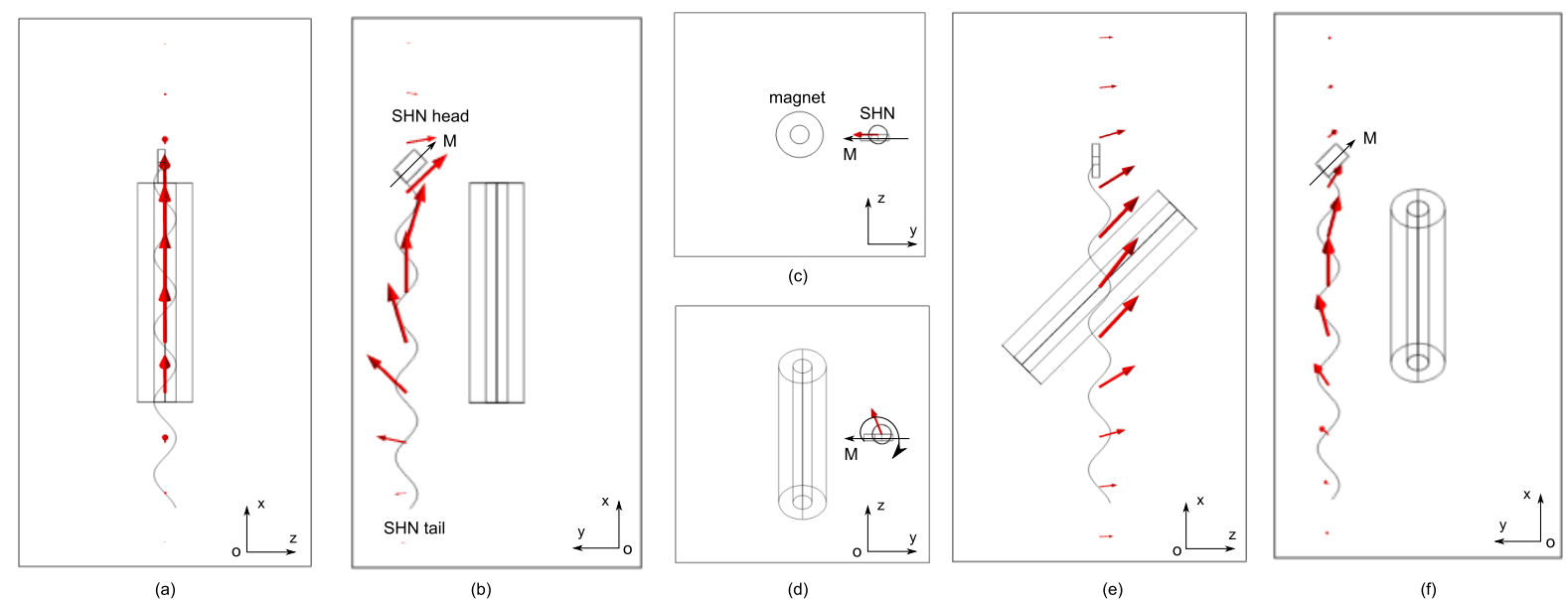

Fig. 6: (a), (b) and (c) The red arrows represent respectively the magnetic field generated by rotating magnet manipulator around the magnetic head at initial position $\left(0^{\circ}\right.$ configuration $)$ in the plane xoz, xoy and yoz. The black arrows represent the magnetization of magnetic head. (e), (f), and (d) represent respectively (a), (b), and (c) with the rotating magnet in a $45^{\circ}$ configuration.

with four different head shapes : SHN10-none (with no head), SHN10-cylinder, SHN10-sphere and SHN10square, are tested in the following experiments.

A SHN10-none showed a cut-off frequency with a brutal stop, which was described in [15]. The SHN10none rotated synchronously with the rotating magnetic manipulator until a maximal rotation frequency, which was defined as a cut-off frequency. Beyond this cutoff frequency, the SHN10-none lost its synchronization with the rotating field, suddenly stopped rotating. The rotational propulsion characteristics of SHN10-none at different Re are depicted by Fig. 8a. The helical swimmers have to overcome the rotational fluidic drag torque, which depends on the viscosity of the liquid and rotation frequency. Beyond the cut-off frequency, the magnetic manipulator can no longer provide enough torque to overcome the fluidic drag torque. The more viscous the liquid is, the bigger the fluidic drag torque is. Thus, the cut-off frequency decreases with the $R e$.

The SHN10-cylinder, SHN10-sphere and SHN10square showed the similar shapes of the rotational propulsion characteristics curves to that of the SHN10none (as shown in Fig. 8a) : synchronization with the rotating field and brutal stop. Fig. 9 summarizes the values of the cut-off frequency of the helical swimmers with magnetic tail and with different heads in function of Re. The SHN10-none have the biggest cutoff frequency values, because the SHN10-none do not have a head to create additional fluidic drag torque. The square head created more drag torque than the spherical and cylindrical heads.

In conclusion, the head shape of a helical swimmer does not influence on the shape of the rotational propulsion characteristics curve, but it influences on the values of the cut-off frequency.

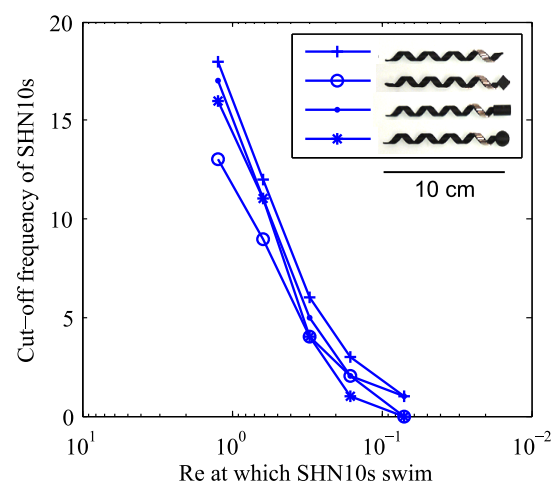

Fig. 9: The maximal rotation frequency of the helical swimmers with magnetic tail and with different heads in function of $R e$.

\section{B. Magnetic positioning influence on rotational propulsion characteristics}

The following experiments are designed in order to compare the rotational propulsion characteristics between a helical swimmer with a magnetic tail and a helical swimmer with a magnetic head. Only a helical swimmer with a square plate head (SHN10magSquare) was used, because it was not easy to cover the spherical and cylindrical head with magnets.

The SHN10-magSquare showed different rotational propulsion characteristics from the SHN10s with magnetic tails. Fig. $8 \mathrm{~b}$ presents the rotational propulsion characteristics of SHN10-magSquare at different Re. The rotation frequency of SHN10-magSquare increased linearly with the rotation frequency of the rotating magnetic field at first. The rotation frequency of the SHN10-magSquare reached a maximum and then decreased if the rotation frequency of the magnetic field increased further. The SHN10-magSquare showed a 


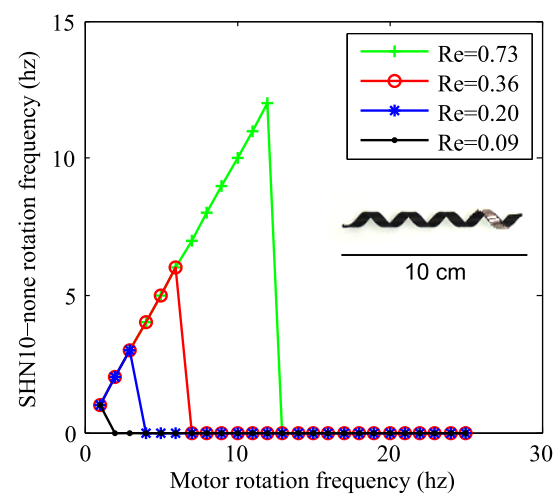

(a)

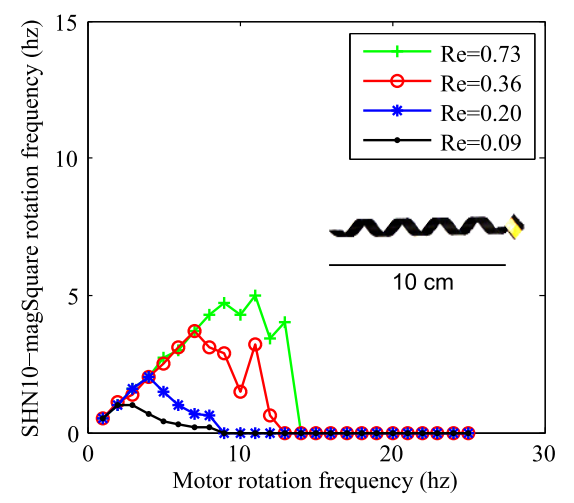

(b)

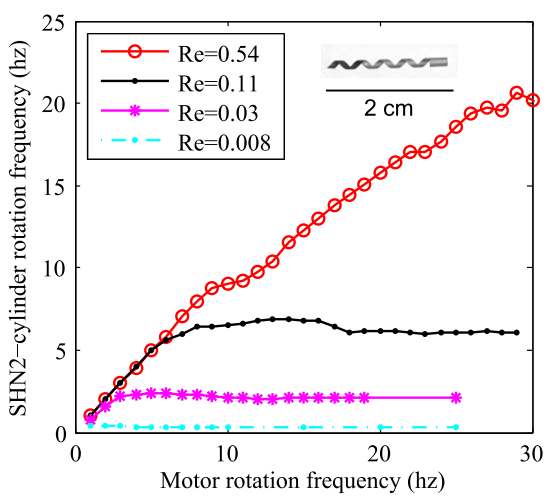

(c)

Fig. 8: (a) The rotation of the SHN10-none was synchronized with the rotating field and showed a cut-off frequency with a brutal stop. (b) The rotation of SHN10-magSquare was not synchronized with the rotating field and showed a cut-off frequency with a gentle decline. (c) The SHN2-cylinder showed a saturation of frequency.

cut-off frequency with a gentle decline. After the cut-off frequency, the decrease of the rotation frequency was not regular. At $R e=0.09$ and $R e=0.20$, the decrease was monotone and almost linear. At $R e=0.36$ and $R e=0.73$, the decrease was not stable. Some rebounds existed, but the overall trend was that the rotation frequency decreased towards zero.

The helical swimmers with magnetic heads have different rotational propulsion characteristics than those of the helical swimmers with magnetic tails. We discuss here the reason which causes this difference. The magnetization direction of the SHN10-none with magnetic tail, which contributes to propelling itself by following the rotating magnetic field, is perpendicular to its axis (M1 in Fig. 5). We defined each possible magnetization direction of the helical swimmer which contributes to propulsion as a magnetization phase. The SHN10-none has several magnetization phases, because its first pitch is covered by 17 magnets. On the opposite, the SHN10-magSquare with a magnetic head has just one magnetization phase, which is in the plane of the magnetic square plate. We presume that this number of magnetization phases influences the rotational propulsion characteristics of the helical swimmer.

\section{Magnetization phase influence on rotational propulsion characteristics}

In order to validate the presumption that rotational propulsion characteristics are influenced by the number of magnetization phases, the following experiments are designed.

The SHN2-cylinder with its helical tail uniformly coated by magnetic materials has much more magnetization phases than the SHN10-none, because the magnetic layer of the later is not continuous. Since the SHN2-cylinder is uniformly coated, its number of magnetization phases is considered as nearing infinity.
The rotational propulsion characteristics of the SHN2cylinder at different $R e$ is shown in Fig. 8c. The rotational propulsion characteristics curve of SHN2-cylinder is different from that of the SHN10s with magnetic tails and SHN10-magSquare. The rotation frequency of the SHN2-cylinder rotated in sync with the rotating magnetic field at the beginning, then the increase rate decreased, and finally the rotation frequency stabilized at a value witch was slightly smaller than its maximum. This value is called as the saturation of frequency of the SHN2-cylinder. The increase step and the saturation frequency of the SHN2-cylinder depend strongly on the Reynolds number. At relatively high $R e(R e=0.54)$, the rotation frequency of the SHN2-cylinder increased continuously, and the saturation frequency was not reached before $25 \mathrm{~Hz}$. At relatively low $\operatorname{Re}(R e=0.008)$, the saturation frequency was reached before $1 \mathrm{~Hz}$. The increase step was not shown.

In conclusion, the number of magnetization phases does influence the rotational propulsion characteristics of the helical swimmers . The rotational propulsion characteristics of the three different helical swimmers with different magnetization phases are different. The SHN10-magSquare with 1 magnetization phase showed a cut-off frequency with a gentle decline towards zero. The SHN10s with magnetic tails with several magnetization phases rotated in sync with the rotating field. However, after it lost the synchronization with the rotating field, it showed a brutal stop. The SHN2cylinder with a magnetic tail uniformly coated having infinity magnetization phases showed a saturation of frequency. The rotation frequency increased with the rotating field, then the increase rate decreased and the rotation frequency stabilized at a value slightly smaller than its maximal rotation frequency. This stable value of rotation frequency is called the saturation of frequency. 
TABLE III: Summary of the rotational propulsion characteristics of different helical swimmers.

\begin{tabular}{|c|c|c|c|}
\hline Model & $\begin{array}{l}\text { Magnetic } \\
\text { part }\end{array}$ & $\begin{array}{l}\text { Number of } \\
\text { magnetization } \\
\text { phases }\end{array}$ & $\begin{array}{l}\text { Rotational } \\
\text { propulsion } \\
\text { characteristics }\end{array}$ \\
\hline SHN10-magSquare & head & 1 & cut-off gentle \\
\hline SHN10-none & tail & several & cut-off brutal \\
\hline SHN10-cylinder & tail & several & cut-off brutal \\
\hline SHN10-sphere & tail & several & cut-off brutal \\
\hline SHN10-square & tail & several & cut-off brutal \\
\hline SHN2-cylinder & tail & infinite & saturation \\
\hline
\end{tabular}

\section{CONCLUSION}

In this paper, we proposed to study the head shapes and magnetic positioning influences on the rotational propulsion characteristics of the helical swimmers with scaled-up experimental system.

Helical swimmers with their tails covered by small magnets showed a cut-off frequency with brutal stop, whatever the head shapes they had. The helical swimmer without a head had the highest value of cutoff frequency, because it had no head to create additional drag torque. The square head created more drag torque than the spherical and cylindrical heads. The head shape does not influence on the rotational propulsion characteristics curve, but on the values of cut-off frequency. A helical swimmer with a magnetic square head showed a cut-off frequency with a gentle decline. A helical swimmer with its helical tail uniformly coated by ferromagnetic material showed a saturation of frequency. Its rotation frequency increased synchronously with the rotating magnetic field, then it stabilized at a value slightly smaller than its maximum. The difference of the three types of rotational propulsion characteristics is caused by the different number the magnetization phases. The magnetization phase is defined as a possible magnetization direction of the helical swimmer which contributes to propulsion. Table III summarizes the number of magnetization phases and the rotational propulsion characteristics of different helical swimmers appeared in this paper.

We expect that a microswimmer with a uniformly coated helical magnetic tail will show a saturation of frequency. In this case, the swimming performances will not decrease after the cut-off frequency. The characterization of the propulsion behaviors of the microswimmers with uniformly coated ferromagnetic helical tails will be validated in future works.

\section{ACKNOWLEDGMENT}

We acknowledge funding from Émergence-UPMC2012 research program. The authors are also grateful to Sinan Haliyo, Sylvain Pledel, Christophe Grand, Thomas Seon, Tianyi Li and Jean ochin Abrahamians for providing technical supports on the experimental set-up.

\section{REFERENCES}

[1] B.J. Nelson, I.K. Kaliakatsos, and J.J. Abbott. Microrobots for minimally invasive medicine. Annual Review of Biomedical Engineering, 12(1):55-85, 2010.

[2] E.M. Purcell. Life at low reynolds number. American Journal of Physics, 45(1) :3-11, 1977.

[3] E.M. Purcell. The efficiency of propulsion by a rotating flagellum. Proc Natl Acad Sci U S A, 94(21):11307-11, 1997.

[4] H.C. Berg and R.A. Anderson. Bacteria swim by rotating their flagellar filaments. Nature, 245(5425) :380-382, 1973.

[5] T. Honda, K.I. Arai, and K. Ishiyama. Micro swimming mechanisms propelled by external magnetic fields. Magnetics, IEEE Transactions on, 32(5) :5085 -5087, sep 1996.

[6] L. Zhang, J.J. Abbott, L. Dong, B.E. Kratochvil, D. Bell, and B.J. Nelson. Artificial bacterial flagella : Fabrication and magnetic control. Appl. Phys. Lett., 94(6), 2009.

[7] L. Zhang, K.E. Peyer, and B.J. Nelson. Artificial bacterial flagella for micromanipulation. Lab Chip, 10 :2203-2215, 2010.

[8] Ambarish Ghosh and Peer Fischer. Controlled propulsion of artificial magnetic nanostructured propellers. Nano Letters, 9(6) :2243-2245, 2009.

[9] G. Hwang, S. Haliyo, and S. Régnier. Remotely powered propulsion of helical nanobelts. In Robotics Science and Systems, 2010.

[10] G. Hwang, R. Braive, L. Couraud, A. Cavanna, O. Abdelkarim, I. Robert-Philip, A. Beveratos, I. Sagnes, S. Haliyo, and S. Régnier. Electro-osmotic propulsion of helical nanobelt swimmers. The International Journal of Robotics Research, 30(7) :806-819, 2011.

[11] S. Tottori, L. Zhang, F. Qiu, K. Krawczyk, A. Franco-Obregn, and B.J. Nelson. Magnetic helical micromachines : Fabrication, controlled swimming, and cargo transport. Advanced Materials, 24(6) :pp. 811-816, February 2012. highlighted as the front cover.

[12] K. Ishiyama, K.I. Arai, M. Sendoh, and A. Yamazaki. Spiraltype micro-machine for medical applications. In Micromechatronics and Human Science, 2000. MHS 2000. Proceedings of 2000 International Symposium on, pages 65-69, 2000.

[13] D.J. Bell, S. Leutenegger, K.M. Hammar, L.X. Dong, and B.J. Nelson. Flagella-like propulsion for microrobots using a nanocoil and a rotating electromagnetic field. In Robotics and Automation, 2007 IEEE International Conference on, pages $1128-1133$, april 2007.

[14] L. Zhang, J.J. Abbott, L. Dong, K.E. Peyer, B.E. Kratochvil, H. Zhang, C. Bergeles, and B. J. Nelson. Characterizing the swimming properties of artificial bacterial flagella. Nano Letters, $9: 3663-3667$, October 2009.

[15] T. Xu, G. Hwang, N. Andreff, and S. Régnier. Scaled-up helical nanobelt modeling and simulation at low reynolds numbers. In ICRA'12 IEEE International Conference on Robotics and Automation, pages $4045-4051,2012$.

[16] O. Reynolds. An experimental investigation of the circumstances which determine whether the motion of water shall be direct or sinuous, and of the law of resistance in parallel channels. Philosophical Transactions of the Royal Society of London, 174 :pp. 935-982, 1883.

[17] J.J. Abbott, M. Cosentino Lagomarsino, L. Zhang, L. Dong, and B.J. Nelson. How should microrobots swim? The International Journal of Robotics Research, 28(11-12) :1434-1447, 2009.

[18] T.W.R. Fountain, P.V. Kailat, and J.J. Abbott. Wireless control of magnetic helical microrobots using a rotating-permanentmagnet manipulator. In Robotics and Automation (ICRA), 2010 IEEE International Conference, pages 576 -581, may 2010.

[19] A.W. Mahoney, D.L. Cowan, K.M. Miller, and J.J. Abbott. Control of untethered magnetically actuated tools using a rotating permanent magnet in any position. In ICRA, pages 33753380. IEEE, 2012.

[20] D. Jiles. Introduction to magnetism and magnetic materials; 2 nd ed. Chapman and Hall, Boca Raton, FL, 1998. 